probably because different workers use different techniques. Adults immunized with type B capsular polysaccharide give encouraging antibody responses, but children under 2 years old may not be capable of responding adequately to this antigen.

\section{Symposium V}

\section{Equipment for haemotology}

Coagulometers A. A. SHARP (Department of Pathology, Radcliffe Infirmary, Oxford) The development of mechanical or optical devices to measure the time of fibrin formation in mixtures of reacting coagulant factors has proceeded with remarkable rapidity over the past two years.

By now eight different types of equipment are known to be available and seven of them have been evaluated, one of which has been withdrawn from the British market. Two other devices appear not to have completed their development.

The devices that have been evaluated may be divided into two main types.

\begin{tabular}{ll}
\hline Mechanical & Optical \\
\cline { 2 - 2 } Mechrolab & Clotek \\
Depex & Coag-a-Pet \\
& Electra 620 \\
& Electra 600 \\
\hline
\end{tabular}

All are basically mechanical stopwatches which have the theoretical advantage of removing the variable of various technician skills. Three are multichannel: Mechrolab (2), Depex (4), Coag-a-Pet (6), while only the Electra 600 offers semi-automation automatically testing and recording 50 consecutive clotting times. Under test, all machines met their manufacturers' claims on the type of tests performed, but they showed a variety of cost/test. Only one showed a true saving of technician time (Electra 600).

The overall results obtained during the evaluation revealed that these machines showed a high degree of reproducibility, differences being due to variations in the volumes of plasma or additions, the former being the most critical. Certain results pointed out limitation of existing techniques.

It has proved impossible to define any ideal machine as any equipment must be available for a given task in a laboratory, bearing in mind the available technician skills.

So far, the availability of servicing and spare parts for certain equipment has not been fully tested, but in one instance, the situation has proved far from satisfactory.
Automatic Platelet Counters R. M. Rowan (Department of Haematology, Royal Infirmary, Glasgow) Platelet enumeration continues to pose problems in the haematology laboratory; however, during the past year, two new platelet counting systems, employing differing cell rating mechanisms, have been introduced.

The Coulter Thrombocounter is semi-automatic, performing counts on platelet-rich plasma, obtained by sedimentation, and has a throughput of 20 samples per hour. The Thrombocounter utilizes an electronic gating principle which counts cells by sensing a change in the electrical conductivity of a channel separating two electrolyte solutions when cells flow through this channel. Since counts are performed on platelet-rich plasma, tables are provided which simultaneously correct for the excess platelets in the plasma and the haematocrit.

The Technicon Platelet Autocounter is fully automatic, handling whole blood at a rate of 40 samples per hour. The Autocounter utilizes a light scattering principle which counts particles by sensing the scattering of light occurring when particles flow through the illuminated sensing chamber of a microoptical system. Platelets and leucocytes are counted by the optical system, the result being presented as analog signals to a single pen recorder. A white cell count, performed independently, must be subtracted from the instrument count to obtain a whole blood platelet count.

Statistical and field evaluations have been carried out on both systems. Reproducibility studies yield excellent results at all levels of platelet count. Carryover and departures from linearity, although statistically significant, are not sufficient to cause concern in routine use. Correlation with microscopy counts is good. Discrepant counting may occur in dysproteinaemic and myeloproliferative states.

Significant improvements in the process of platelet enumeration are achieved by these systems.

Automatic Coombs Test Washers R. N. IBBOTson (Blood Transfusion Centre, Birmingham) A comparative evaluation of three red cell washing machines currently available has been carried out. These include the Dade, Sorvall CW1/AF1, and Spectra Auto I machines. Investigations cover aspects of the mechanical specifications, cell washing facilities, efficiency and bacteriological safety of the machines. All three machines have the capability for automatic triple washing of red cells in saline, the complete cycle times being just over six minutes in the Dade and Sorvall and 14 minutes with the Spectra. The Spectra automatically adds the antihuman globulin reagent and completes a spin type Coombs test. Triple washing of red cells suspended 
in serum containing $8.8 \mathrm{~g} / 100 \mathrm{ml}$ of gamma globulin resulted in a residual protein content of 41,38 , and $85 \mu \mathrm{g} / \mathrm{ml}$ respectively in the Dade, Sorvall, and Spectra machines and compares with a residue of $44 \mu \mathrm{g} / \mathrm{ml}$ using standard manual techniques in $75 \times 12 \mathrm{~mm}$ size test tubes. A fourth red cell wash is practicable on the Dade and Sorvall machines but not with the Spectra.

No contamination by aerosol was found using a bacterial suspension of Chromobacterium violaceum on agar plates attached to the outsides of the machines. Bench contamination is possible from drips from the lid of the Dade and from a bowl drain of the Sorvall models.

Interface for Coulter S Counters M. G. NELSON (Department of Pathology, Royal Victoria Hospital, Belfast) In the Department of Haematology, Royal Victoria Hospital, Belfast, punch cards have been used since 1965 to process the laboratory data and a number of systems have been evaluated.

The present system involved the replacement of the multichannel analyser by a Coulter $S$, the development of an interface and the use of a computer to handle punch cards in an off-line batch mode. The specially designed silent interface with transistorized circuitry captures the output from the Coulter S, visualizes the results, automatically prints the data on a continuous paper roll, and transmits the information to a card punch unit located in a data processing room. Further requested test data and the PID are manually added to the punch card. The completed deck of cards is input on a dedicated laboratory computer to format report documents, produce ward listings and quality $\stackrel{0}{\overrightarrow{0}}$ control information. The laboratory data are stored $\underline{\underline{0}}$ for one month on a computer disc and the punch $\stackrel{\Rightarrow}{\Rightarrow}$ cards are retained as hard copy for one year. This $\stackrel{0}{9}$ system has been in routine daily use for almost two $\bar{C}$ years and has proved both reliable and flexible. Many initial problems have been eliminated and the $\frac{0}{\circ}$ computer programmes have been debugged. The $\stackrel{\Phi}{\Omega}$ whole system has recently been improved by an advancement which interleaves batches of haematology cards onto the computer when it is being used 'on-line' by another laboratory department.

A stand alone 'Reporter 7' system which does not require computer facilities has been developed. The interface in this system has a solid state memory î which permits the output of the Coulter $S$ to be reproduced in duplicate by an IBM input/output $\overrightarrow{.}$ writer on both a laboratory report slip and con- $\vec{\circ}$ tinuous stationery. This can then be used as both a worksheet and a storage document. The Reporter $7 ?$ embodies signals to indicate results which are 'out of range' and samples which require further dilution for leucocyte counting.

Computer Linkage for Technicon SMA 4A D. 금 ROBERTSON SMITH (Department of Pathology, Royal $\stackrel{\mathbb{Q}}{-}$ Berkshire Hospital, Reading) At the Royal Berlo $\overrightarrow{0}$ shire Hospital, the Technicon SMA 4A analyser linked on-line to a digital PDP 12 computer as pa. of an automated haematological and biochemica laboratory. The method of operation for profile screening and routine work is described, together with the production of ward reports and cumulative reports. 